\title{
BLOW-UP FOR A DEGENERATE DIFFUSION PROBLEM NOT IN DIVERGENCE FORM
}

\author{
RAÚL FERREIRA, ARTURO DE PABLO, AND JULIO D. ROSSI
}

\begin{abstract}
We study the behaviour of solutions of the nonlinear diffusion equation in the half-line, $\mathbb{R}_{+}=(0, \infty)$, with a nonlinear boundary condition,

$$
\begin{cases}u_{t}=u u_{x x}, & (x, t) \in \mathbb{R}_{+} \times(0, T), \\ -u_{x}(0, t)=u^{p}(0, t), & t \in(0, T), \\ u(x, 0)=u_{0}(x), & x \in \mathbb{R}_{+},\end{cases}
$$

with $p>0$. We describe in terms of $p$ and the initial datum when the solution is global in time and when it blows-up in finite time. For blowing up solutions we find the blow-up rate and the blow-up set and we describe the asymptotic behaviour close to the blow-up time in terms of a self-similar profile. The stationary character of the support is proved both for global solutions and blowing-up solutions. Also we obtain results for the problem in a bounded interval.
\end{abstract}

\section{INTRODUCTION AND MAIN RESULTS}

In this paper we deal with the problem

$$
\begin{cases}u_{t}=u u_{x x}, & (x, t) \in \mathbb{R}_{+} \times(0, T), \\ -u_{x}(0, t)=u^{p}(0, t), & t \in(0, T), \\ u(x, 0)=u_{0}(x), & x \in \mathbb{R}_{+},\end{cases}
$$

with $p>0$. We assume that $u_{0}$ is a non-trivial, continuous, non-increasing and compactly supported function. We also consider in the last section the problem posed in a bounded interval $[0, L] \subset \mathbb{R}_{+}$with a zero flux boundary condition on the right border, $u_{x}(L, t)=0$.

Problem (1.1) can be thought of as a model for nonlinear heat propagation. In this case $u$ stands for the temperature and the boundary condition represents a nonlinear radiation law. This kind of boundary conditions appear also in combustion problems when the reaction happens only at the boundary of the container, for example because of the presence of a solid catalyzer, see [21] for a justification.

Local in time existence of weak solutions, defined in the usual integral way, can be easily established as for instance in [6], see Section 2. In fact, classical theory implies that the solution is smooth when positive. On the contrary it is only Lipschitz at the boundary of the support. The time $T$ is the maximal existence time for the solution, which may be finite or infinite. If $T<\infty$, then $u$ becomes unbounded in finite time and we say that it blows up. If $T=\infty$ we say that the solution is global.

In this article we are interested in the blow-up phenomenon, a subject that has deserved a great deal of attention in recent years, see for example the book [23] and

1991 Mathematics Subject Classification. 35B40, 35B35, 35K65.

Key words and phrases. Blow-up, asymptotic behaviour, nonlinear boundary conditions. 
the surveys $[7,20]$. For specific references about blow-up in problems with nonlinear boundary conditions see $[4,10]$.

Problem (1.1) can be viewed as a limit case for the family of problems

$$
\begin{cases}u_{t}=u^{r} u_{x x}, & (x, t) \in \mathbb{R}_{+} \times(0, T), \\ -u_{x}(0, t)=u^{p}(0, t), & t \in(0, T), \\ u(x, 0)=u_{0}(x), & x \in \mathbb{R}_{+},\end{cases}
$$

with $p>0$ and $r \in \mathbb{R}$. If $r \neq 1$ we can write problem (1.2) in divergence form by means of the change of variables $v=u^{1-r} /|1-r|$, thus getting the problem

$$
\begin{cases}v_{t}=\left(v^{m-1} v_{x}\right)_{x}, & (x, t) \in \mathbb{R}_{+} \times(0, T), \\ -c v^{m-1} v_{x}(0, t)=v^{q}(0, t), & t \in(0, T), \\ v(x, 0)=v_{0}(x), & x \in \mathbb{R}_{+},\end{cases}
$$

with $m=1 /(1-r), q=p m, c=|m|^{q-m} m$ and $v_{0}=u_{0}^{1-r} /|1-r|$. If $r<1$, we have $m>0, q>0, c>0$, and problem (1.3) is the well known porous medium equation (the classical heat equation if $r=0$, the fast diffusion equation if $r<0$ ) with reaction. The blow-up phenomenon for this problems has been extensively studied in the works $[8,11,12,14,17,22]$, both for the half-line or a bounded interval. If on the contrary $r>1$, we obtain $m<0, q<0$ and $c<0$. We are thus in presence of superfast diffusion with a singular absorption, which produces a phenomenon known as quenching; only the case $q=m$ in a bounded interval has been studied, see $[9]$.

Also related with our problem are the works $[6,16,24]$ on the problem

$$
\begin{cases}u_{t}=u\left(\Delta u+u^{p}\right), & (x, t) \in \Omega \times(0, T), \\ u=0, & (x, t) \in \partial \Omega \times(0, T), \\ u(x, 0)=u_{0}(x), & x \in \Omega,\end{cases}
$$

where the reaction is produced at the interior of the domain $\Omega \subset \mathbb{R}^{n}$. The blow-up in those examples depends on the exponent $p$ but also on the initial datum in some cases, and even on the domain if $p=1$.

Our first aim is to study when the solutions to problem (1.1) are globally defined or they blow up in finite time. The picture depends obviously on $p$, but also crucially on the initial data. In fact, there always exist global solutions for every $p>0$, contrary to what occurs for problem (1.2) with $r<1$, where every solution blows up if $(2-r) / 2<p \leq 2-r$ independently of the initial condition, see $[8,14]$. In the sequel we assume that $u_{0}$ has compact support $[0, N]$. We prove that the critical global existence exponent for problem (1.1) is $p=1$ in the following sense:

Theorem 1.1. Let $\operatorname{supp}\left(u_{0}\right)=[0, N]$. We have,

i) if $p<1$ the solution is global;

ii) if $p=1$ the solution is global if and only if $N \leq 1$;

iii) assume $p>1$; if $u_{0}(x) \leq A\left(1-A^{p-1} x\right)_{+}$for some $A>0$ then the solution is global; if $u_{0}(0)>N^{-1 /(p-1)}$ then the solution blows up.

Once we have characterized for which exponents the solution to problem (1.1) can or cannot blow up, we want to study the way the blowing up solutions behave as approaching the blow-up time. This means that we must investigate where the solutions blow up (the blow-up set), the speed at which they blow up (the blow-up rate) and the shape of the solutions close to the blow-up time (the blow-up profile). 
To this purpose we consider from now on exponents $p \geq 1$ and $u$ a blowing up solution with blow-up time $T$.

We begin with the blow-up rate of blowing up solutions.

Theorem 1.2. Let $u$ be a solution of (1.1) with finite blow-up time T. As t approaches $T$ we have

$$
\|u(\cdot, t)\|_{\infty} \sim(T-t)^{-1 /(2 p-1)},
$$

where $f \sim g$ means that there exist finite positive constants $c_{1}, c_{2}$ such that $c_{1} g \leq$ $f \leq c_{2} g$.

We continue the analysis of problem (1.1) by studying the asymptotic behaviour of blowing up solutions. As is often the case in nonlinear problems of parabolic type, the characteristic properties of an equation, in this case the blow-up behaviour, are displayed by means of appropriate self-similar solutions, see for instance [3]. In our case there also exist self-similar solutions for every $p \geq 1$ (even explicit if $p=1$ ) see Theorem 2.1, and they in fact reflect the asymptotic behaviour of blowing up solutions. They take the form

$$
U(x, t)=(T-t)^{-\alpha} G(\xi), \quad \xi=x(T-t)^{-\beta},
$$

with the similarity exponents $\alpha=1 /(2 p-1)$ and $\beta=(p-1) \alpha$. Observe that in the blow-up range we have $\alpha>0, \beta \geq 0$. The existence of self-similar profiles is settled in the next section. The problem satisfied by the self-similar profile $G$ is

$$
\left\{\begin{array}{l}
G G^{\prime \prime}-\alpha G-\beta \xi G^{\prime}=0, \quad \xi>0, \\
-G^{\prime}(0)=G^{p}(0) .
\end{array}\right.
$$

Let $u$ be a solution to problem (1.1) that blows up in time $T$, satisfying the rate (1.4), and change variables as,

$$
g(\xi, \tau)=(T-t)^{\alpha} u(x, t), \quad \xi=x(T-t)^{-\beta}, \quad \tau=-\log (1-t / T) .
$$

For each initial value $u_{0}$ we define the so-called $\omega$-limit set of $u_{0}$ as the set of possible limits of the corresponding rescaled orbits $g$ :

$$
\begin{aligned}
\omega\left(u_{0}\right)=\{\quad & G \in C\left(\mathbb{R}_{+}\right), G \geq 0: \exists \tau_{j} \rightarrow \infty \text { such that } \\
& \left.\lim _{\tau_{j} \rightarrow \infty} g\left(\cdot, \tau_{j}\right)=G \text { uniformly in compact sets of } \mathbb{R}_{+}\right\} .
\end{aligned}
$$

Theorem 1.3. The $\omega$-limit set of any initial value $u_{0}$ for which the solution $u$ blows up at time $T$ is contained in the set of non-trivial solutions of the problem (1.6), that is, the set of self-similar profiles.

Also of interest is to study the propagation properties of the solutions, i.e., the way the support of $u(\cdot, t)$ behaves. In particular we prove

Theorem 1.4. The support of $u(\cdot, t)$ is constant in time.

This is in great contrast with problem (1.2) with $0<r<1$, where the support of the solutions always expands. If $r \leq 0$ it expands with infinite velocity.

Next we study the blow-up set, which is defined in the following way,

$$
B(u)=\left\{x \in[0, \infty): \exists x_{n} \rightarrow x, t_{n} \nearrow T, \text { with } u\left(x_{n}, t_{n}\right) \rightarrow+\infty\right\} .
$$

We have single-point blow-up if $p>1$ and regional blow-up if $p=1$. 
Theorem 1.5. Let $u$ be a blowing up solution of (1.1). Then

i) if $p>1$ the blow-up set is $B(u)=\{0\}$.

ii) if $p=1$ the blow-up set verifies $[0,1] \subset B(u) \subset[0, \min \{N, 2\}]$.

Our theorem is again in contrast with the case $0<r<1$ in problem (1.2), where the blow-up set is a single point if $p>1$, but it is the whole half-line if $p<1$ and a bounded interval if $p=1$. Notice that in the case of problem (1.1), in order to have blowing up solutions we need $p \geq 1$.

As for the problem in a bounded interval, we perform at the end of the paper the same analysis as for the previous problem. We say in advance that the critical exponent is different: here every solution blows up independently of the initial data for every $p>0$. The rate is the same if $p \geq 1, \alpha=1 /(2 p-1)$, but it is $\alpha=1 / p$ if $0<p<1$.

Organization of the paper. In Section 2 we present some tools used in the rest of the paper, including the construction of the self-similar profiles, Theorem 2.1. In Section 3 we prove Theorem 1.1 and Theorem 1.2. Theorem 1.3 is proved in Section 4 as well as Theorems 1.4 and 1.5 are proved in Section 5. We devote the last section to treat the case of a bounded interval.

\section{Preliminaires}

Fix $T>0$, put $Q_{T}=\mathbb{R}_{+} \times[0, T]$ and consider the space $V=L^{\infty}\left(Q_{T}\right) \cap$ $L^{2}\left(0, T ; H^{1}\left(\mathbb{R}_{+}\right)\right)$.

Definition 2.1. We say that $u \in V$ is a weak solution to problem (1.1) if the following equality holds for every test function $\varphi \in V$ vanishing for large $x$,

$$
\begin{aligned}
\iint_{Q_{T}}\left(u \varphi_{t}-u_{x}(u \varphi)_{x}\right) d x d t & +\int_{0}^{T} u^{p+1}(0, t) \varphi(0, t) d t \\
& =\int_{0}^{\infty}\left(u(x, T) \varphi(x, T)-u_{0}(x) \varphi(x, 0)\right) d x .
\end{aligned}
$$

Following the same arguments used in [6] we have the following results. We refer to that paper for the details. Observe that in our case the reaction term is localized at $x=0$ where the solution is strictly positive; therefore the reaction function is smooth.

Lemma 2.1. If $u$ is a nonnegative weak solution then for every $0<r<1$ we have

$$
\iint_{Q_{T}} \frac{\left|u_{x}\right|^{2}}{u^{r}} d x d t \leq C(r, T)
$$

In particular, $u$ is Lipschitz continuous in space.

Proposition 2.1. There exists a unique weak solution of problem (1.1) which is smooth in its positivity set. Moreover, a comparison principle holds.

Proposition 2.2. If $u$ is a weak solution of problem (1.1) in $0<x<R(t)$ satisfying $u(R(t), t)=0$, then the function $v$ defined as $v(x, t)=u(x, t)$ if $0<x \leq R(t)$, $v(x, t)=0$ if $x>R(t)$, is a weak solution in $\mathbb{R}_{+}$.

Proposition 2.2 allows problem (1.1) to have always stationary solutions.

Proposition 2.3. If $p>0$ problem (1.1) admits the family of stationary solutions

$$
z_{A}(x)=A\left(1-A^{p-1} x\right)_{+} \quad A>0 .
$$


Remark that when $A$ increases the support of $z_{A}$ increases if $p<1$, decreases if $p>1$ and is constant $[0,1]$ if $p=1$.

Problem (1.1) admits also self-similar solutions, i.e. solutions in the form (1.5), with profile satisfying (1.6).

Theorem 2.1. Problem (1.6) admits bounded solutions if and only if $p \geq 1$. If $p=1$ the family of solutions is explicit, all of them having compact support:

$$
G_{R}(\xi)=\left\{\begin{array}{cl}
\frac{1}{2} \xi^{2}-R \xi+R & \text { if } 0 \leq \xi \leq \xi_{R} \\
0 & \text { if } \xi \geq \xi_{R}
\end{array}\right.
$$

where $\xi_{R}=R-\sqrt{R^{2}-2 R}, R \geq 2$. If $p>1$ the solution is unique, positive, strictly decreasing and it satisfies

$$
G(\xi) \sim \xi^{-1 /(p-1)} \quad \text { as } \xi \rightarrow \infty .
$$

The precise decay (2.3) in the case $p>1$ is crucial. Other decays would lead either to a self-similar solution with global blow-up (something which is impossible) or to a trivial asymptotic profile. Observe also that the support of the profile $G_{R}$ in (2.2) in the case $p=1$ shrinks as $R$ increases, and has limits [0,2] and $[0,1]$ as $R$ tends to 2 or infinity, respectively.

The construction of the profiles is based in the following lemma.

Lemma 2.2. Let $\alpha>0, \beta \in \mathbb{R}, V \in \mathbb{R}$, and consider the problem

$$
\left\{\begin{array}{l}
F F^{\prime \prime}-\alpha F-\beta \xi F^{\prime}=0 \quad \xi \in \mathbb{R}_{+}, \\
F(0)=1 \\
-F^{\prime}(0)=V
\end{array}\right.
$$

If $\beta<0$ there is no bounded solution. If $\beta=0$, for every $V \geq \sqrt{2 \alpha}$ there exists a unique bounded solution, which has compact support, and no bounded solution exists if $0<V<\sqrt{2 \alpha}$. If $\beta>0$, there exists a unique value $V=V_{*}>0$ such that the problem has a bounded solution, which is unique, strictly decreasing and satisfies the decay rate (2.3).

The existence of self-similar profiles for our problem is now immediate.

Proof of Theorem 2.1. Let $F_{1}$ be a solution to problem (2.4) with $\alpha=1 /(2 p-1)$ and $\beta=(p-1) \alpha$. Then the scaled function $F_{\lambda}(\xi)=\lambda^{2} F_{1}(\xi / \lambda)$ satisfies the equation for the profile plus the boundary conditions $F_{\lambda}(0)=\lambda^{2},-F_{\lambda}^{\prime}(0)=\lambda V$. Therefore it suffices to choose $G=F_{\lambda}$ with $\lambda=V^{1 /(2 p-1)}$.

Proof of Lemma 2.2. The case $\beta=0$ can be integrated directly $\left(F^{\prime \prime}=\alpha\right)$, thus obtaining the explicit (unbounded) parabola $F(\xi)=1-V \xi+\alpha \xi^{2} / 2$. If $V \geq \sqrt{2 \alpha}$, and thanks to Proposition 2.2, we can consider the bounded solution obtained extending $F$ by zero for $\xi>\left(V-\sqrt{V^{2}-2 \alpha}\right) / \alpha$.

For the general case $\beta \neq 0$ we introduce the variables

$$
X=\frac{\xi F^{\prime}}{F}, \quad Y=\frac{\xi^{2}}{F}, \quad \eta=\log \xi .
$$


This kind of transformation goes back to $[2,19]$, and is used for instance in $[8,18]$. The resulting system is

$$
\left\{\begin{array}{l}
\frac{d X}{d \eta}=X(1-X)+Y(\alpha+\beta X), \\
\frac{d Y}{d \eta}=Y(2-X) .
\end{array}\right.
$$

Assume $\beta>0$. We look for nonnegative profiles $F$, so we consider only the upper plane $\{Y>0\}$. As we are interested in solutions with $F(0)=1$ and $F^{\prime}(0)$ finite, the orbits we are looking for start at the critical point $A=(0,0)$. The local analysis of this point is straightforward.

Proposition 2.4. The linearization of (2.6) around $A=(0,0)$ has matrix $\left(\begin{array}{ll}1 & \alpha \\ 0 & 2\end{array}\right)$ with eigenvalues $\lambda_{1}=1$ and $\lambda_{2}=2$ and corresponding eigenvectors $e_{1}=(1,0)$ and $e_{2}=(\alpha, 1)$. Thus $A$ is a repeler.

To study the point at infinity in this phase-plane, we perform the inversion change of variables $H=1 / Y$. We arrive at

$$
\left\{\begin{array}{l}
\frac{d X}{d \eta}=X(1-m X)+\frac{\alpha+\beta X}{H}, \\
\frac{d H}{d \eta}=-H(2-X) .
\end{array}\right.
$$

In order to eliminate the singularity we perform the nonlinear change of variable given implicitly by

$$
\frac{d \eta}{d \tau}=H(\eta)
$$

Observe that this change preserves the direction of the flow on the upper half-plane $\{H>0\}$, which is the same, $\{Y>0\}$. Then $X, H$ satisfy

$$
\left\{\begin{array}{l}
\frac{d X}{d \tau}=H X(1-X)+(\alpha+\beta X), \\
\frac{d H}{d \tau}=-H^{2}(2-X) .
\end{array}\right.
$$

The proper behaviour (2.3) in these variables corresponds to the critical point $B=(-\alpha / \beta, 0)$. The local analysis around this point is again straightforward.

Proposition 2.5. The critical point $B=(-\alpha / \beta, 0)$ is a saddle-node. The linearization of (2.7) around $B=(-\alpha / \beta, 0)$ has matrix $\left(\begin{array}{cc}\beta & -\frac{\alpha(\alpha+\beta)}{\beta^{2}} \\ 0 & 0\end{array}\right)$ with eigenvalues $\lambda_{1}=\beta$ and $\lambda_{2}=0$ and corresponding eigenvectors $e_{1}=(1,0)$ and $e_{2}=$ $\left(1, \beta^{3} /(\alpha(\alpha+\beta))\right)$. The point $B$ is a repeler on the half-plane $\{H<0\}$ and a saddle on the half-plane $\{H>0\}$.

Existence of the connection. We are looking for an orbit connecting the critical points $A$ and $B$. As there is a unique orbit $\sigma_{*}$ arriving at $B$, we just have to trace back where it comes from. In the $X Y$-plane the critical point $B$ corresponds to $(-\alpha / \beta,+\infty)$. We observe that $d Y / d \eta>0$ for every $X<0, Y>0, d X / d \eta<0$ for $X<-\alpha / \beta$ and that $d X / d \eta>0$ for $X=0, Y>0$. Then the orbit $\sigma_{*}$ necessarily comes from $A$. 


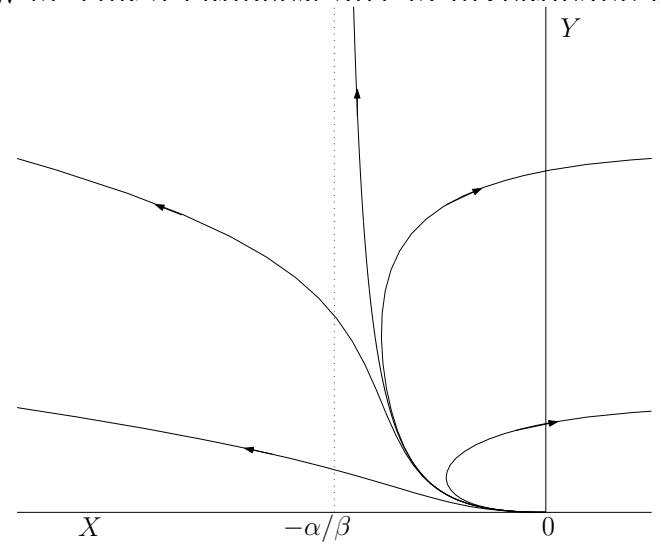

Figure 1. The trajectories in the $X Y$-plane for $p>1$.

We now have to look more carefully at the behaviour of this trajectory near the point $A$. From Proposition 2.4, we have that in the second quadrant, $\{X<0, Y>$ $0\}$, all the trajectories exit the origin tangent to the horizontal axis. Moreover, it is easy to check that they do this quadratically. In particular our trajectory exits $A$ like $Y \sim \Lambda X^{2}$ for some $\Lambda>0$. This gives the value $V_{*}=1 / \sqrt{\Lambda}$. Even more, $X<0$ implies that the profile $F_{*}$ corresponding to the orbit $\sigma_{*}$ is strictly decreasing.

In order to give a complete understanding of the picture in the upper plane $\{Y>0\}$, and prove that $\sigma_{*}$ gives the unique profile, we consider all the trajectories starting at the origin $(X, Y)=(0,0)$ and check that other choices of $V$ give profiles that are either unbounded or defined only in a bounded interval.

First of all, from the equation of the profile (2.4), it is easy to see that once the function $F$ satisfies $F^{\prime}\left(\xi_{0}\right) \geq 0$ for some $\xi_{0} \geq 0$, then $F^{\prime}(\xi)>0$ for every $\xi>\xi_{0}$, and the profile becomes unbounded. This corresponds to the trajectories in the $X Y$-plane that exit the origin in the first quadrant $(V \leq 0)$ and also those in the second quadrant that cross the vertical axis $\left(0<V<V_{*}\right)$.

We also observe that the trajectory $\sigma_{*}$ is a separatrix in the second quadrant between those trajectories that cross the vertical axis and those trajectories that cross the vertical line $X=-\alpha / \beta$. We now prove that these last trajectories, which correspond to taking $V>V_{*}$, give profiles that vanish at a finite value of $\xi$, thus not being defined in the whole $\mathbb{R}_{+}$. To see this let $Y=Y(X)$ be a trajectory passing through a point $(-\alpha / \beta, D), D>0$, at a time $\eta_{0} \in \mathbb{R}$. The first equation in (2.6) gives, for $\eta>\eta_{0}$,

$$
\frac{d X}{d \eta} \leq-X^{2}
$$

since $\alpha+\beta X<0, Y>0$. This implies that there exists a finite $\eta_{\infty}$ such that

$$
\lim _{\eta \rightarrow \eta_{\infty}} X(\eta)=-\infty
$$

This also implies $\lim _{\eta \rightarrow \eta_{\infty}} Y(\eta)=Y_{\infty}>0$. We obtain a profile $F$ with compact support $\left[0, \xi_{\infty}\right], \xi_{\infty}=\mathrm{e}^{\eta_{\infty}}$. By Lemma 2.1 we have that $F^{\prime}\left(\xi_{\infty}\right)$ is finite. This

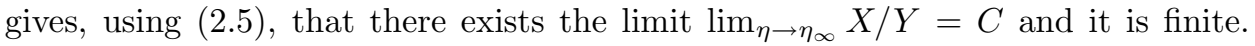
But now (2.6) and L'Hôpital's rule gives

$$
C=\lim _{\eta \rightarrow \eta_{\infty}} \frac{d X}{d Y}=\lim _{\eta \rightarrow \eta_{\infty}} \frac{-X^{2}+\beta X Y}{-X Y}=C-\beta,
$$


and we arrive at a contradiction. These profiles have $F^{\prime}\left(\xi_{\infty}\right)=-\infty$ and will be useful in comparison arguments.

If $\beta<0$ the critical point at infinity $(-\alpha / \beta, \infty)$ has moved to the first quadrant, and every profile is unbounded since the orbits cross the vertical axis.

\section{Global existence versus Blow-up}

First section we characterize when the solutions to problem (1.1) are global in time or they blow up.

Proof of Theorem 1.1. $i$ ) If $p<1$ we can use as a supersolution the function $z_{A}$ of (2.1) with $A$ large, so by comparison every solution is bounded.

ii) If $p=1$ and $N \leq 1$, the function $z_{A}(x)=A(1-x)$ also works as a supersolution, and hence $u$ is bounded. If $N>1$ we use comparison with the self-similar blowing-up solution $U(x, t)=\left(T_{0}-t\right)^{-1} G_{R}(x)$, the profile $G_{R}$ given in (2.2). If $R$ and $T_{0}$ are large we can get $u_{0}(x) \geq U(x, 0)$. Thus $u$ blows up at time less than $T_{0}$.

iii) The condition for global existence is obvious, as seen in the previous cases. If now $u_{0}(0)>A>N^{-1 /(p-1)}$ and $u_{0}^{\prime \prime} \geq 0$, we compare from below with a function of the form

$$
W(x, t)=\left(T_{0}-t\right)^{-1} \lambda^{-2} G_{R}(\lambda x),
$$

for some $T_{0}, \lambda$ and $R$ properly chosen, and with $G_{R}$ given in (2.2). First of all we observe that $W$ is a solution to the problem

$$
\begin{cases}W_{t}=W W_{x x} & (x, t) \in \mathbb{R}_{+} \times\left(0, T_{0}\right), \\ -W_{x}(0, t)=\lambda W(0, t) & t \in\left(0, T_{0}\right), \\ W(x, 0)=T_{0}^{-1} \lambda^{-2} G_{R}(\lambda x) & x \in \mathbb{R}_{+},\end{cases}
$$

On the other hand, since $u_{t}$ is nonnegative, $u$ is a supersolution to this problem if we choose $\lambda=A^{p-1}$ and provided we can compare the initial values. This is done by taking $R$ large enough in order to get $1<\xi_{R}<N A^{p-1}$ (see the comment below Theorem 2.1) and then $T_{0}$ large. Observe that the support of $W$ is $\left[0, \xi_{R} / \lambda\right] \subsetneq[0, N]$. Thus $u$ blows up before $T_{0}$. For the general case we consider a convex initial function below $u_{0}$ satisfying the required conditions. Comparison finishes the proof.

For positive initial values, $u_{0}>0$, we only prove

Theorem 3.1. Assume $u_{0}(x)>0$ for every $x \geq 0$. Then for problem (1.1), i) if $p \leq 1 / 2$ the solution is global;

ii) if $p \geq 1$ the solution blows up.

Proof. $i$ ) We compare with a self-similar solution $w$ to the problem with $p=1 / 2$ in the form $w(x, t)=e^{r t} G\left(x e^{-s t}\right)$. The existence of such solution can be obtained in a completely analogous way as in the proof of Theorem 2.1. This function $w$ is a supersolution to our problem and is globally defined.

ii) This case follows easily from comparison with a blowing up subsolution with large support, see Theorem 1.1.

Contrary to what occurs for compactly supported solutions, the global solutions are not globally bounded if they are positive.

Proposition 3.1. Let $p<1$. If $u_{0}>0$ and $u$ is global then it is unbounded. 
Proof. Assume by contradiction that there exists a constant $K>0$ such that $u(0, t) \leq K$ for every $t>0$. We use the same argument than in the proof of Theorem 1.1, iii). Consider the function (3.1) with any $R \geq 2$, which is a solution to problem (3.2). If we take $\lambda=K^{p-1}$, and noting that necessarily $p<1$ if $u$ is global, then $u$ is a supersolution to that problem (provided $T_{0}$ is large in order to compare the initial values), and comparison implies that $u$ must blow up.

We now concentrate in obtaining estimates of the growth of the solutions, i.e., we obtain the blow-up rates.

Proof of Theorem 1.2. The main ingredient in this proof is the use of the intersection comparison principle: given two solutions of the equation, the number of intersections does not increase in the interior of the domain as time evolves.

For a solution $u$ with blow-up time $T$ we will use the self-similar function,

$$
W(x, t)=(T-t)^{-\alpha} G\left(x(T-t)^{-\beta}\right),
$$

where $G$ is a solution of (1.6) in its support. These profiles $G$ were constructed in the previous section.

Let us begin with the lower estimate. We consider $W$ given by (3.3) with $G$ an unbounded profile such that $W(x, 0)=T^{-\alpha} G\left(x T^{-\beta}\right)$ has only one intersection with $u_{0}(x)$ with $W(0,0)<u_{0}(0)$. This can be achieved taking $G(0)$ small enough. The number of intersections does not increase. In fact, the Hopf Lemma implies that no new intersection may appear at $x=0$. Since $G$ is unbounded and $u$ is compactly supported no new intersection can neither appear at infinity. Therefore, the number of intersections between $u(x, t)$ and $W(x, t)$ is at most one. If we have no intersections, $u(x, t) \supsetneqq W(x, t)$ for $t \geq t_{0}$, the strong maximum principle implies that $u(x, t)<W(x, t)$ for $t>t_{0}$ hence both functions can not blow up at the same time $T$. This contradiction implies that the number of intersections is always one, hence $u(0, t)>W(0, t)=c(T-t)^{-\alpha}$ for all $t \in(0, T)$.

To obtain the upper estimate we proceed in a similar way but in this case we consider a self-similar solution with $G(0)$ large. This gives us a decreasing selfsimilar profile with compact support $\left[0, \xi_{0}\right]$. Recall that for $p>1$ we have $G^{\prime}\left(\xi_{0}\right)=$ $-\infty$ and hence $W$ is not a solution in the whole $\mathbb{R}_{+}$. We then consider the problem in $\left[0, \xi_{0}(T-t)^{\beta}\right] \times(0, T)$ with Dirichlet boundary condition on the right end. Since $G(0)$ is large we may assume that $W(x, 0)$ and $u_{0}(x)$ have only one intersection with $W(0,0)>u_{0}(0)$. Remark that at the right boundary $\xi_{0}(T-t)^{\beta}$ the solution $u$ is strictly positive, therefore no new intersection may appear there. As before, we can conclude that the number of intersections is at most one. The fact that $u(x, t)$ is a supersolution of the above Dirichlet problem implies that the number of intersections is always one. This gives $u(0, t)<W(0, t)=C(T-t)^{-\alpha}$ for all $t \in(0, T)$.

We end this section with an estimate of the blow-up time in the case $p=1$. It is based on the fact that the self-similar profiles have compact support and they are even explicit.

Proposition 3.2. Let $p=1$ and $G_{R}$ any self-similar profile given by (2.2), then we have

$$
\inf _{x>0} \frac{G_{R}(x)}{u_{0}(x)} \leq T \leq \sup _{x>0} \frac{G_{R}(x)}{u_{0}(x)}
$$


Proof. To prove the upper estimate, assume $\hat{T}=\sup _{x>0} G_{R}(x) / u_{0}(x)<\infty$. Otherwise the estimate holds trivially and is meaningless. By comparison, $u(x, t) \geq$ $(\hat{T}-t)^{-1} G_{R}(x)$, which implies $T \leq \hat{T}$. The estimate from below follows in the same way.

\section{Asymptotic Behaviour}

Proof of Theorem 1.3. Recall that for a solution $u$ that blows up in time $T$ we put

$$
g(\xi, \tau)=(T-t)^{\alpha} u(x, t), \quad \xi=x(T-t)^{-\beta}, \quad \tau=-\log (1-t / T) .
$$

This rescaled function $g$ verifies

$$
\begin{cases}g_{\tau}=g g_{\xi \xi}-\alpha g-\beta \xi g_{\xi}, & (\xi, \tau) \in \mathbb{R}_{+} \times \mathbb{R}_{+}, \\ -g_{\xi}(0, \tau)=g^{p}(0, \tau), & \tau \in \mathbb{R}_{+}, \\ g(\xi, 0)=T^{-\alpha} u_{0}\left(T^{\beta} \xi\right), & \xi \in \mathbb{R}_{+} .\end{cases}
$$

Thanks to the blow-up rate (1.4) we know that $g$ is bounded. The behaviour of $u$ near $t=T$ is translated into the behaviour of $g$ as $\tau \rightarrow \infty$.

It is easy to see that there exists a sequence $\tau_{j} \rightarrow \infty$ such that

$$
\lim _{j \rightarrow \infty} g\left(\xi, \tau+\tau_{j}\right)=g_{*}(\xi, \tau)
$$

uniformly in compact sets of $\mathbb{R}_{+}$. We want to prove that the function $g_{*}$ does not depend on $\tau$ and therefore it coincides with a stationary solution $G$ constructed in Section 2 .

First, let us consider the case $p=1$, i.e., $\beta=0$. In this case the convergence follows from the existence of the explicit Lyapunov functional

$$
L_{g}(\tau)=\int_{0}^{\infty}\left(\frac{1}{2}\left(g_{\xi}\right)^{2}(\xi, \tau)+g(\xi, \tau)\right) d \xi-\frac{1}{2} g^{2}(0, \tau),
$$

which is decreasing along the orbits,

$$
\frac{d L_{g}}{d \tau}(\tau)=-4 \int_{0}^{\infty}\left(\left(g^{1 / 2}\right)_{\tau}\right)^{2}(\xi, \tau) d \xi \leq 0,
$$

and bounded from below (thanks to the blow-up rates (1.4)). Therefore we conclude in a rather standard way the convergence of the orbits to a stationary solution, see for instance $[1,13]$. Indeed,

$$
\begin{aligned}
\left\|g^{1 / 2}\left(\cdot, \tau_{j}+\tau\right)-g^{1 / 2}\left(\cdot, \tau_{j}\right)\right\|_{L^{2}([0, \infty])}^{2} & =\int_{0}^{\infty}\left|g^{1 / 2}\left(\xi, \tau_{j}+\tau\right)-g^{1 / 2}\left(\xi, \tau_{j}\right)\right|^{2} d \xi \\
& \leq \tau \int_{0}^{\infty} \int_{\tau_{j}}^{\tau_{j}+\tau}\left|\left(g^{1 / 2}\right)_{\tau}\right|^{2}(\xi, s) d s d \xi \rightarrow 0
\end{aligned}
$$

as $j \rightarrow \infty$, uniformly for bounded $\tau$. Therefore, the sequence $g^{1 / 2}\left(\xi, \tau_{j}+\tau\right)$ converges in the space $L^{\infty}\left([0, \tau]: L^{2}([0, \infty))\right)$ for every $\tau>0$. The limit does not depend on $\tau$ and is a stationary solution of $(4.2)$ in $[0, \infty)$ and hence it is one of the stationary profiles found in Section 2.

If now $p>1$ we construct a Lyapunov function for $g$ following the ideas of $[13,25]$, taking note of the boundary condition. See those papers for the details, specially for the regularization needed to justify the formal calculations. We write the equation for $g$ in (4.2) in the following way

$$
g_{\tau}=g\left(g_{\xi \xi}+b\left(\xi, g, g_{\xi}\right)\right),
$$


where $b(\xi, g, z)=-\beta \xi z / g-\alpha$. Consider the function

$$
L_{g}(\tau)=\int_{0}^{\infty} \Phi\left(\xi, g(\xi, \tau), g_{\xi}(\xi, \tau)\right) d \xi-\frac{1}{p+1} g^{p+1}(0, \tau) .
$$

Differentiating and integrating by parts, we get

$$
\begin{aligned}
\frac{d}{d \tau} L_{g}(\tau) & =-\left(g^{p}(0, \tau)+\Phi_{z}\left(0, g, g_{\xi}\right)\right) g_{\tau}(0, \tau) \\
& -\int_{0}^{\infty} \frac{1}{g} \Phi_{z z}\left(g_{\tau}\right)^{2} d \xi+\int_{0}^{\infty}\left(\Phi_{g}-\Phi_{\xi z}-\Phi_{g z} g_{\xi}+b \Phi_{z z}\right) g_{\tau} d \xi
\end{aligned}
$$

We can eliminate the last integral by choosing appropriately the function $\Phi$ using the method of characteristics. It is given formally by

$$
\Phi(\xi, g, z)=\int_{0}^{z}(z-s) \rho(\xi, g, s) d s-\int_{0}^{g} b(\xi, s, 0) \rho(\xi, s, 0) d s,
$$

where $\rho(\xi, g, z)=\exp \left(\int_{0}^{\xi} b_{z}(\eta) d \eta\right)$, and $b_{z}$ is evaluated along the characteristic $\phi(\eta, \xi, g, z)$, solution to

$$
\left\{\begin{array}{l}
\phi^{\prime \prime}+b\left(\eta, \phi, \phi^{\prime}\right)=0 \quad 0<\eta<\xi \\
\phi(\xi)=g, \phi^{\prime}(\xi)=z
\end{array}\right.
$$

Thus, from the study performed in Section 2 we know that there exists a unique solution which is decreasing for every $g>0, z \leq 0$, but there is no solution if $g=0$. Let us define the function $\rho$ as

$$
\rho(\xi, g, z)= \begin{cases}\exp \left(-\beta \int_{0}^{\xi} \eta \phi^{-1}(\eta, \xi, g, z) d \eta\right), & \text { if } g>0 \\ 0, & \text { if } g=0\end{cases}
$$

It is then clear that whenever $\beta>0$, as in our case, we have $\rho \leq 1$. A lower estimate for $\rho$ is crucial in our Lyapunov argument. The lower bound that we have is

$$
\rho(\xi, g, z) \geq e^{-\beta \xi^{2} /(2 g)} .
$$

We now calculate

$$
\Phi_{z}\left(0, g, g_{\xi}\right)=\int_{0}^{g_{\xi}} \rho(0, g, s) d s=g_{\xi}(0, \tau)=-g^{p}(0, \tau) .
$$

Putting all together we get

$$
\frac{d}{d \tau} L_{g}(\tau)=-\int_{0}^{\infty} \frac{1}{g} \rho\left(\xi, g, g_{\xi}\right)\left(g_{\tau}\right)^{2} d \xi
$$

Since $b(\xi, g, 0) \leq 0$ and the function $g$ is bounded, we have that $L_{g}(\tau) \geq-C$.

On the other hand, we claim that if we restrict ourselves to $0<\xi<R$ for any given $R>0$, we get $g \geq C(R)>0$ and thus $\rho \geq C(R)>0$. Assume this claim. The previous estimates imply

$$
\begin{aligned}
4 \int_{\tau_{1}}^{\tau_{2}} \int_{0}^{R}\left(\left(g^{1 / 2}\right)_{\tau}\right)^{2} d \xi d \tau & \leq \frac{1}{C(R)} \int_{\tau_{1}}^{\tau_{2}} \int_{0}^{\infty} \frac{1}{g} \rho\left(\xi, g, g_{\xi}\right)\left(g_{\tau}\right)^{2} d \xi d \tau \\
& =\frac{1}{C(R)}\left(L_{g}\left(\tau_{1}\right)-L_{g}\left(\tau_{2}\right)\right) \leq C
\end{aligned}
$$

We conclude as for the case $p=1$ the convergence of the orbits to a stationary solution in $[0, R]$. Hence

$$
\lim _{\tau \rightarrow \infty} g(\xi, \tau)=G(\xi), \quad \text { in }[0, R],
$$


where $G$ is a stationary profile, decreasing and positive in $[0, R]$.

In order to finish the proof we only have to prove the claim and to extend the above convergence to the whole $[0,+\infty)$.

To do this, consider the following problem

$$
\begin{cases}w_{\tau}=w w_{\xi \xi}-\alpha w, & (\xi, \tau) \in \mathbb{R}_{+} \times \mathbb{R}_{+}, \\ w(0, \tau)=c, & \tau \in \mathbb{R}_{+}, \\ w(\xi, 0)=w_{0}(\xi), & \xi \in \mathbb{R}_{+} .\end{cases}
$$

First of all, the lower bound for the blow-up rate gives that $g(\xi, \tau)$ is a supersolution if $w_{0}$ and $c$ are small enough. On the other hand, the function $w(\xi)=(a-\alpha \xi / 2)_{+}^{2}$ is a subsolution if $a$ is small. A comparison argument gives $g(\xi, \tau) \geq w(\xi)$. Therefore we obtain a lower bound for $g(\xi, \tau)$ in $[0, a / \alpha]$. Thanks to our previous arguments we obtain the desired convergence to a positive profile in $[0, a / \alpha]$. Let us extend it to the whole $\mathbb{R}_{+}$. Define $R^{*}=\sup \{R>0$, (4.8) holds $\}$. Assume that $R^{*}<\infty$. It is easy to see that $G\left(R^{*}\right)>0$ and thus $g(\xi, \tau)>0$ for every $\xi \in\left[R^{*}, R^{*}+\delta\right]$ for some $\delta>0$ and $\tau$ large. This implies that we can obtain the lower estimate of $\rho$ in the set $\left[0, R^{*}+\delta\right]$ proving the convergence to a stationary profile in $\left[0, R^{*}+\delta\right]$. A contradiction that proves that $R^{*}=\infty$. Finally, the limit profile is the unique positive profile obtained in Section 2 .

\section{Propagation of the support. Blow-up sets}

In this section we obtain the blow-up sets and study the (no) evolution of the support.

Proof of Theorem 1.4. The fact that the support of the solution does not shrink follows from comparison with any solution $v$ that has initial data $v_{0} \leq u_{0}$ with the same support as $u_{0}$, convex and compatible with the boundary conditions. Observe that $v$ remains convex and hence nondecreasing in time.

To prove that the support does not increase we observe the following easy fact

Proposition 5.1. Take any point $0<x_{*}<N$. Let $K>0$ be such that $u\left(x_{*}, t\right) \leq$ $K$ for every $t_{1} \leq t \leq t_{2}$, then $\operatorname{supp}(u(\cdot, t)) \subset[0, N]$ for every $t_{1} \leq t \leq t_{2}$.

Proof: We use as comparison the function $P(x)=K^{\prime}(N-x)_{+} /\left(N-x_{*}\right)$, for some $K^{\prime}>K$ large enough. It is a supersolution to the problem

$$
\begin{cases}w_{t}=w w_{x x}, & x>x_{*}, t \in\left(t_{1}, t_{2}\right), \\ w\left(x_{*}, t\right)=K, & t \in\left(t_{1}, t_{2}\right), \\ w\left(x, t_{1}\right)=u\left(x, t_{1}\right), & x>x_{*},\end{cases}
$$

as well as $u$ is a subsolution.

Proof of Theorem 1.5. i) Using the estimate from above in the blow rate (1.4), we can compare $u$ with the solution $w$ to the peaking problem

$$
\begin{cases}w_{t}=\frac{1}{2}\left(w^{2}\right)_{x x}, & (x, t) \in \mathbb{R}_{+} \times(0, T), \\ w(0, t)=c(T-t)^{-1 /(2 p-1)}, & t \in(0, T), \\ w(x, 0)=u_{0}(x), & x \in \mathbb{R}_{+},\end{cases}
$$

This problem has been considered in the work [15], in which the authors prove single-point blow-up if $p>1$.

ii) The fact that in the case $p=1$ the blow-up set $B(u)$ contains the interval $[0,1]$ comes from the fact that every self-similar profile is positive in $[0,1]$ together with the convergence result, Theorem 1.3. We also know, Theorem 1.4 , that $B(u) \subset$ 
$[0, N]$. Finally, to prove that $B(u) \subset[0,2]$, we can use again the convergence result plus the fact that all the self-similar solutions have support contained in $[0,2]$. Then we know that $g(x, \tau)$ goes to zero for every $x>2$. We now can apply the technique developed in [5], used also in [9], to show that for $x>2$ we have the decay $g(x, \tau) \leq C \mathrm{e}^{-\tau}$, that is, $u(x, t) \leq C$.

\section{The PRoblem In A Bounded INTERVAL}

We consider in this section the problem

$$
\begin{cases}u_{t}=u u_{x x}, & (x, t) \in(0, L) \times(0, T), \\ -u_{x}(0, t)=u^{p}(0, t), & t \in(0, T), \\ -u_{x}(L, t)=0, & t \in(0, T), \\ u(x, 0)=u_{0}(x), & x \in[0, L],\end{cases}
$$

with $p>0$ and continuous initial datum $u_{0}(x) \geq \delta>0$. The maximum principle implies $u(x, t) \geq \delta$. Observe that if $u_{0}(L)=0$, Theorem 1.4 implies that problem (6.1) reduces to problem (1.1).

Theorem 6.1. The solution to problem (6.1) always blows up for every $p>0$ and every initial value.

Proof. The proof follows three steps. We first show that the solution is unbounded. Next, we consider the case $0<p<1$ and a convex, decreasing, compatible with the boundary conditions initial data. Finally we deal with the general case by comparison.

Integrating the equation $u_{t} / u=u_{x x}$ we get

$$
\frac{d}{d t} \int_{0}^{L} \log u(x, t) d x=u^{p}(0, t) \geq \delta^{p}>0 .
$$

Therefore $u$ is not bounded. Let us now consider the case $0<p<1$, and assume by the previous calculation that $u(0, t)>1$. Integrating by parts the equation in (6.1) we get, for $M(t)=\int_{0}^{L} u(x, t) d x$,

$$
\begin{aligned}
M^{\prime}(t) & =u^{p+1}(0, t)-\int_{0}^{L}\left(u_{x}\right)^{2}(x, t) d x \\
& \geq u^{p+1}(0, t)-u^{2 p}(0, t) L \geq C u^{p+1}(0, t) .
\end{aligned}
$$

On the other hand, if $u_{0}^{\prime \prime} \geq 0$ we have $u_{x x} \geq 0$ and then, by the Mean Value Theorem, we obtain

$$
u(x, t) \geq u(0, t)\left(1-u^{p-1}(0, t) x\right)_{+}
$$

for every $x, t>0$. This estimate gives, if $0<p<1$, the equivalences

$$
u(x, t) \sim u(0, t) \sim M(t)
$$

for every $0 \leq x \leq L$ and $t>0$. This implies $M^{\prime}(t) \geq C M^{p+1}(t)$, which means that $M(t)$ blows up in finite time, and so does $u$. Observe that for any strictly positive initial data we may consider a convex decreasing function below it. A comparison argument gives blow-up for positive initial data for $0<p<1$. The case $p \geq 1$ follows immediately by comparison, since solutions to the problem (6.1) with $p_{1}$ are supersolutions to the problem with a different power $p_{2}<p_{1}$, provided $u(0, t)>1$. 
As in the case of the half-line we characterize the self-similar blowing up solutions in the case $p=1$. Let $H_{R}$ be the parabola, if $0<R \leq 2$,

$$
H_{R}(x)=\frac{1}{2} x^{2}-R x+R,
$$

and $\widetilde{H}_{R}$ the truncated parabola (see $(2.2)$ ), if $R \geq 2$,

$$
\widetilde{H}_{R}(x)=\left\{\begin{array}{cl}
\frac{1}{2} x^{2}-R x+R & \text { if } 0 \leq x \leq x_{R} \\
0 & \text { if } x \geq x_{R}
\end{array}\right.
$$

where $x_{R}=R-\sqrt{R^{2}-2 R}$. We have that problem (6.1) admits the family of solutions

$$
U(x, t)=(T-t)^{-1} G(x)
$$

The profiles are:

i) if $0<L \leq 1$, then $G=H_{L}$ is the only profile;

ii) if $1<L<2$, then $G=H_{L}$ and also $G=\widetilde{H}_{R}$ with $R>\frac{L^{2}}{2(L-1)}$;

iii) if $L \geq 2$, then $G=\widetilde{H}_{R}$ with $R \geq 2$.

Theorem 6.2. Let $u$ be a solution to problem (6.1) which blows up at time $T$.

i) If $0<p<1$ and $u_{0}^{\prime \prime} \geq 0$, then $\|u(\cdot, t)\|_{\infty} \sim C(T-t)^{-1 / p}$. ii) If $p \geq 1$, then $\|u(\cdot, t)\|_{\infty} \sim c(T-t)^{-1 /(2 p-1)}$.

Proof. $i$ ) The case $0<p<1$ follows from the proof of the previous theorem, since we have in fact, instead of $(6.2), M^{\prime}(t) \sim M^{p+1}(t)$. This gives the rate $M(t) \sim(T-t)^{-1 / p}$, and (6.4) implies the rate for $u$.

ii) Let $\alpha=1 /(2 p-1)$ and $\beta=(p-1) \alpha$. Let us consider, as in the proof of Theorem 1.2, a self similar solution $W(x, t)=(T-t)^{-\alpha} G\left(x(T-t)^{-\beta}\right)$ with $G(0)$ small. The initial data $u_{0}$ and $W(x, 0)$ have at most one intersection. As before this implies that $u(0, t) \geq W(0, t)=c(T-t)^{-\alpha}$.

The proof of the upper bound is exactly the same as the one of Theorem 1.2. In this case we only have to observe that $u$ and $W$ may have one or zero intersections. If the number of intersections is zero, it may increase by one from $x=L$. The rest of the proof follows exactly as before.

As a consequence of the above results we obtain global blow-up for $0<p<1$. If $p \geq 1$ the blow-up set can be characterized as in the case of the half-line.

Theorem 6.3. Let $u$ be a blowing up solution of (6.1) with $u_{0}$ convex. Then

i) if $p>1$ the blow-up set is $B(u)=\{0\}$.

ii) if $p=1$ the blow-up set verifies $[0, \min \{1, L\}] \subset B(u) \subset[0, \min \{2, L\}]$.

iii) if $0<p<1$ and $u_{0}^{\prime \prime} \geq 0$ the blow-up set is $B(u)=[0, L]$.

Proof. The first two assertions follows as in Theorem 1.5. The last one comes from (6.3).

\section{Acknowledgements}

R. Ferreira and A. de Pablo partially supported by DGICYT grant BFM200204572 (Spain). J. D. Rossi supported by Universidad de Buenos Aires under grant EX066, CONICET (Argentina) and Fundacion Antorchas (Argentina). This research was performed while the third author (JDR) was a visitor at Universidad Carlos III de Madrid. He is extremely grateful to this institution for its hospitality. 


\section{REFERENCES}

[1] D. G. Aronson, M. G. Crandall, and L. A. Peletier, Stabilization of solutions of a degenerate nonlinear diffusion problem, Nonlinear Anal 16 (1982), 1001-1022.

[2] G. I. Barenblatt, On some unsteady motions of a liquid or a gas in a porous medium, Prikl. Mat. Mekh. 16 (1952), 67-78, (in Russian).

[3] Scaling, Self-Similarity and Intermediate Asymptotics, Cambridge University Press, Cambridge, U.K, 1996.

[4] M. Chlebík and M. Fila, Some recent results on the blow-up on the boundary for the heat equation, Banach Center Publ. 52 (2000), 61-71.

[5] C. Cortazár, M. del Pino, and M. Elgueta, On the blow-up set for $u_{t}=\Delta u^{m}+u^{m}, m>1$, Indiana Univ. Math. J. 47 (1998), 541-561.

[6] R. Dal Passo and S. Luckaus, A degenerate diffusion problem not in divergence form, J. Differential Eqns. 69 (1987), 1-14.

[7] K. Deng and H. Levine, The role of critical exponents in blow-up theorems: the sequel, J. Math. Anal. Appl. 243 (2000), 85-126.

[8] R. Ferreira, A. de Pablo, F. Quiros, and J. D. Rossi, The blow-up profile for a fast diffusion equation with a nonlinear boundary condition, Rocky Mountain J. Math. 33 (2003), 123-146.

[9] _ Superfast quenching, J. Differential Eqns. 199 (2004), 189-209.

[10] M. Fila and J. Filo, Blow-up on the boundary: A survey, Singularities and Differential Equations, Banach Center Publ. 33 (1996), 67-78.

[11] M. Fila and P. Quittner, The blowup rate for the heat equation with a nonlinear boundary condition, Math. Methods Appl. Sci. 14 (1991), 197-205.

[12] J. Filo, Diffusivity versus absorption through the boundary, J. Differential Eqns. 99 (1992), 281-305.

[13] V. A. Galaktionov, On asymptotic self-similar behaviour for a quasilinear heat equation. Single point blow-up, SIAM J. Math. Anal. 26 (1995), 675-693.

[14] V. A. Galaktionov and H. A. Levine, On critical Fujita exponents for heat equations with nonlinear flux boundary conditions on the boundary, Israel J. Math. 94 (1996), 125-146.

[15] B. H. Gilding and M. A. Herrero, Localization and blow-up of thermal waves in nonlinear heat conduction with peaking, Math. Ann. 282 (1998), 223-242.

[16] J. S. Guo, Y. J. Guo, and J. Ch. Tsai, Single-point blow-up patterns for a nonlinear parabolic equation, Nonlinear Anal. TMA 53 (2003), 1149-1165.

[17] B. Hu and H. M. Yin, The profile near blow-up time for solutions of the heat equation with a nonlinear boundary condition, Trans. Amer. Math. Soc. 346 (1994), 117-135.

[18] J. Hulshof, Similarity solutions of the porous medium equation with sign changes, J. Math. Anal. Appl. 157 (1991), 75-111.

[19] C. W. Jones, On reducible nonlinear differential equations occurring in mechanics, Proc. Roy. Soc. 217 (1953), 327-343.

[20] H. A. Levine, The role of critical exponents in blow up theorems, SIAM Rev. 32 (1990), $262-288$.

[21] F. J. Mancebo and J. M. Vega, A model of porous catalyst accounting for incipiently nonisothermal effects, J. Differential Eqns. 151 (1999), 79-110.

[22] A. de Pablo, F. Quiros, and J. D. Rossi, Asymptotic simplification for a reaction-diffusion problem with a nonlinear boundary condition, IMA J. Appl. Math. 67 (2002), 69-98.

[23] A. A. Samarskii, V. A. Galaktionov, S. P. Kurdyumov, and A. P. Mikhailov, Blow-up in problems for quasilinear parabolic equations, Nauka, Moscow, 1987, (in Russian). English transl.: Walter de Gruyter, Berlin, 1995.

[24] M. Ughi, A degenerate parabolic equation modelling the spread of an epidemic, Ann. Mat. Pura Appl 143 (1986), 385-400.

[25] T I. Zelenyak, Stabilization of solution of boundary value problems for a second-order parabolic equation with one space variable, Differ. Uravn. 4 (1986), 34-45, (in Russian), English transl.: Differential Eqns. 4 (1986), 17-22. 
Departamento de Matemáticas, U. Carlos III de Madrid, 28911 Leganés, Spain., eMAIL: raul.ferreira@uc3m.es

Departamento de Matemáticas, U. Carlos ili de Madrid, 28911 Leganés, Spain., eMAIL: arturop@math.uc3m.es

Departamento de Matemática, F.C.E y N., UBA, (1428) Buenos Aires, Argentina., E-MAIL: jrossi@dm.uba.ar 